Explicit motor sequence learning with the paretic arm after stroke

Melanie K Fleming ${ }^{1}{ }^{*}$, Di J Newham ${ }^{1}$ and John C Rothwell ${ }^{2}$.

${ }^{1}$ Centre of Human and Aerospace Physiological Sciences, King's College London, London, $U K$

${ }^{2}$ Institute of Neurology, University College London, London, UK

* Corresponding author

Melanie Fleming

Centre of Human and Aerospace Physiological Sciences

Faculty of Life Sciences and Medicine

King's College London

3.11 Shepherd's House, Guy's Campus, London SE1 1UL, UK

Email: melanie.fleming@kcl.ac.uk

Ph. +44 (0) 2078486679

Fax: +44 (0) 2078486325

Word count: 3703 


\section{Explicit motor sequence learning with the paretic arm after stroke}

\section{Abstract}

Purpose: Motor sequence learning is important for stroke recovery, but experimental tasks require dexterous movements, which are impossible for people with upper limb impairment. This makes it difficult to draw conclusions about the impact of stroke on learning motor sequences. We aimed to test a paradigm requiring gross arm movements to determine whether stroke survivors with upper limb impairment were capable of learning a movement sequence as effectively as age-matched controls.

Materials and Methods: In this case-control study, 12 stroke survivors (10-138 months post-stroke, mean age 64 years) attempted the task once using their affected arm. Ten healthy controls (mean 66 years) used their non-dominant arm. A sequence of 10 movements was repeated 25 times. The variables were: time from target illumination until the cursor left the central square (onset time; OT), accuracy (path length) and movement speed.

Results: OT reduced with training $(\mathrm{p}<0.05)$ for both groups, with no change in movement speed or accuracy ( $>>0.1)$. We quantified learning as the OT difference between the end of training and a random sequence; this was smaller for stroke survivors than controls $(\mathrm{p}=0.015)$.

Conclusions: Stroke survivors can learn a movement sequence with their paretic arm, but demonstrate impairments in sequence specific learning.

Key words: Motor sequence learning, Stroke, Upper Limb, Impairment. 


\section{Introduction}

Following neurological injury to the motor system, such as stroke, the central nervous system has to adapt to the loss of permanently damaged connections and optimise use of remaining connections to regain function. This relearning of motor skills involves the engagement of a network of brain regions [1-6] to restore or compensate for lost patterns of control.

One important component of successful motor control is the ability to learn sequential movement patterns. Indeed, following stroke this is the basis for functional rehabilitation strategies such as task specific training [7]. Studies of sequential motor tasks in stroke survivors have produced inconsistent results with some showing impairment in learning [810] whereas others have found that learning is intact $[11,12]$. However, many of these studies have focussed on tasks using the 'unaffected' rather than the paretic limb, despite the fact that re-learning during physical rehabilitation is mostly focused on the paretic limb. This may be, at least partly, because the traditional sequence learning tasks require dexterous finger movements which are impossible for many stroke survivors with upper limb impairment.

Motor sequence learning tasks can be either "implicit" or "explicit”. For implicit learning tasks the participant is not told of the presence of a repeating pattern of movements whereas for explicit learning they are informed in advance. Reaction times are measured as an indication of the ability to prepare movement in advance of a signal. In both explicit and implicit learning tasks the reaction times become quicker over a number of repetitions. This improvement is due to a combination of non-specific effects, such as familiarity with the apparatus, plus sequence specific effects that allow better motor preparation for the next movement. If a random sequence is introduced after performing the repeated sequence, 
reaction times increase and the difference between the two is taken as a measure of sequencespecific learning $[13,14]$.

Implicit learning with the "unaffected" hand has been found to be altered after stroke $[9,15]$ but motor sequence learning with the affected hand needs further investigation. One study [16] demonstrated that stroke survivors were capable of learning a key-press sequence with their affected hand, but all scored at least 61 (out of 66) on the Fugl-Meyer upper limb assessment (FM) [17] so their level of impairment was extremely mild. If motor sequence learning after stroke is to be better understood it is necessary to use a paradigm that enables stroke survivors with a range of functional impairments to use their paretic arm.

Such tasks have been devised in the past. Dovern et al. [18] used a task requiring large button presses instead of individual keys, but only tested stroke survivors using their "unaffected" hand. In the present experiments we aimed to assess motor sequence learning with the paretic arm of both mild and moderately impaired stroke survivors (defined as a Fugl-Meyer upper limb impairment score of $19-60$ [19]). We therefore used a task that required gross movements of the hand/arm. This explicit learning task involved using a computer mouse to reach for targets on a monitor that illuminated in a repeated order. We examined whether a group of stroke survivors with upper limb impairment would be able to learn the task with their paretic arm as effectively as healthy age-matched participants.

\section{Materials and Methods}

\section{Study design}


This was a case-control study to assess the ability of stroke survivors and healthy agematched controls to learn a movement sequence. All assessments were conducted by a trained neurophysiologist (MKF).

\section{Participants}

Twelve chronic stroke survivors (10 male, mean age 64 years, range 39 - 80; Table 1) and 10 healthy adults ( 6 male, mean age 66 years, range 50 - 85) attempted one session of the motor sequence learning task. Inclusion criteria for the stroke survivors were; aged > 18 years, single stroke $>3$ months duration, unilateral upper limb weakness with a Fugl-Meyer upper limb assessment [17] score $\leq 60$ and no history of other neurological diseases. All but one (\#11, Table 1) of the stroke survivors were right handed prior to the stroke and all used their affected hand to perform the task. Participants were not undergoing any interventions or rehabilitation at the time of participation in this study. Healthy adults denied any history of stroke or other neurological disease, were right handed (mean laterality quotient $87 \%$ ) [20] and used their left hand to perform the task.

This experiment was approved by the local Research Ethics Committee and participants provided written informed consent.

\section{Insert table 1 around here}

\section{Paradigm}

Participants sat at a table $\sim 60 \mathrm{~cm}$ in front of a computer monitor showing four grey circular targets $(2.3 \mathrm{~cm}$ diameter $)$ and a red central square $\left(10.9 \mathrm{~cm}^{-2}\right.$; figure. 1$)$. The circular targets were all equidistant from the central square $(8.5 \mathrm{~cm})$. The number of targets was consistent 
with the traditional sequence learning paradigms which involve four stimulus cues.

Participants held a computer mouse which had been modified by removing the buttons. The programme was run by a custom designed Matlab (The Mathworks Inc., Massachusetts, USA) programme which allowed input of an Excel file (Microsoft 2007) which specified the sequence order. Sequences of 10 movements were specified for these experiments, consistent with previous sequence learning studies $[13,21]$.

Stroke survivors completed the task using their paretic hand. All reported that they did not routinely use this hand to control a computer mouse. Healthy adults used their non-dominant (left) hand to ensure that the use of the computer mouse was novel and considered to be difficult. Participants initially moved the computer mouse to direct the cursor to the central square. One of the circular targets would illuminate (changing from grey to white) $0.3 \mathrm{~s}$ after the cursor entered the central square, indicating that they should move the mouse to direct the cursor into the illuminated target. Once the cursor reached the target it had to remain there for $0.4 \mathrm{~s}$ before the target would return to grey, indicating that they should return to the central square for illumination of the next target. This dwell time at the target was to ensure that participants purposefully and accurately moved the cursor into the target, rather than sliding the cursor through it.

\section{Insert figure 1 around here}

Participants first completed two or three practice sequences to familiarise them with the movement of the mouse to the targets. This was necessary as participants did not routinely use the tested hand to control a computer mouse. They were then informed that they would perform 25 repetitions of a sequence involving 10 movements, and that they should anticipate 
target appearance if they knew which would be next (i.e. explicit learning). Participants were given short breaks between repetitions throughout the test if required. The sequence for each participant was chosen randomly from a pool of eight sequences. Targets were not numbered but if numbers were assigned left to right then examples of the sequences include 1-2-2-4-31-3-4-1-2 and 2-3-3-1-4-2-3-1-3-4. Pilot experiments indicated no noticeable differences in difficulty between the sequences. Following completion of the 25 repetitions participants were instructed to perform a random sequence in order to distinguish between general learning and sequence specific learning effects.

\section{Variables}

Values for onset time (OT, s), movement time (MT, s) and path length (PL, pixels) were automatically computed by the programme and saved into an Excel file. OT was calculated as the time from target illumination to when the cursor left the central square. Since there was a delay of $0.3 \mathrm{~s}$ between the cursor entering the square and target illumination, it was possible to achieve a negative OT if participants anticipated the next target and moved the cursor towards it prior to illumination. MT was the time from the cursor leaving the central square to arriving in the illuminated target. PL was the number of pixels the cursor travelled through to get from the central square to the illuminated target, and as such an increased PL represents reduced accuracy of movement. Speed of cursor movement (pixels.s ${ }^{-1}$ ) was calculated manually by dividing PL by MT.

\section{Analysis}

The median values for OT, PL and speed were calculated for each of the 25 repetitions. The median was used, rather than the mean, as it is less likely to be skewed by outliers when only 10 values are recorded for each repetition. Values were then averaged across consecutive 
repetitions (2-3, $4-5$ etc.) forming 13 blocks. Herein the term "block" refers to these average values.

Statistical analysis was performed using SPSS 21.0 (IBM). Normality was assessed using Shapiro Wilk tests and visual inspection of frequency histograms. If the assumption of normality was sustained $(\mathrm{p}>0.05)$ then parametric statistics were utilised, but otherwise if transformation was ineffective then non-parametric statistics were used. Violations of the sphericity assumption were corrected using the Greenhouse-Geisser correction if Epsilon < 0.75 and the Huyn-Feldt correction if Epsilon > 0.75. Post-hoc pairwise comparisons were conducted with a modified Bonferroni correction [22]. Results are expressed as mean \pm standard error of the mean (SEM) unless otherwise stated. Significance is $\mathrm{p}<0.05$.

One way repeated measures analysis of variance (rmANOVA) or Friedman tests were used to test for the effect of block on OT, PL and speed for each group separately. To test for sequence specific learning effects, planned comparisons using paired samples t-tests or Wilcoxon Signed Rank tests were conducted comparing values from the random sequence with those of the last block of the repeated sequence.

To determine whether baseline task performance differed between groups, independent sample t-tests were used to compare the initial values for OT, PL and speed. To compare learning between groups the OT values were normalised to the first repetition of the repeated sequence and a Mann-Whitney U test used to compare the OT difference between the random sequence and the last block of the repeated sequence (sequence specific learning).

\section{Results}




\section{Age-matched controls}

The OT reduced over the repeated blocks (main effect of block: $F_{3.18,28.62}=12.141, \mathrm{p}<$ 0.001), indicating improvements in the time to react to target illumination with training of the repeated movement sequence. The OT for the last block of the repeated sequence $(0.078 \pm$ $0.06 \mathrm{~s})$ was significantly lower than the subsequent random sequence $(0.421 \pm 0.03 \mathrm{~s} ; \mathrm{t}(9)=-$ $6.601, \mathrm{p}<0.001$ ) indicating that there was significant sequence-specific learning (figure 2).

Neither PL, nor movement speed, changed significantly over the repeated blocks (effect of block: $p>0.3)$. There were no significant differences between the last repeated block and the following random sequence for either variable $(\mathrm{p}>0.2)$.

Insert figure 2 around here

\section{Stroke Survivors}

Patient demographics are presented in table 1. Two of the stroke participants (\#9 and \#10) were unable to complete the task. Participant \#9 found it too difficult to keep the mouse on the table while moving it and the cursor did not move properly on the screen, and participant \#10 found it too tiring to concentrate on the screen (due to poor eyesight). Their data was not used for analysis.

The OT reduced over the repeated blocks (main effect of block: $F_{3.212,28.97}=4.560, p=0.009$ ) indicating improvements in the time to react to target illumination with training of the repeated sequence. Learning was specific to the trained movement sequence, as the OT for 
the last repeated block $(0.33 \pm 0.07 \mathrm{~s})$ was significantly lower than the OT for the subsequent random sequence $(0.50 \pm 0.04 \mathrm{~s} ; \mathrm{t}(9)=-2.882, \mathrm{p}=0.018$, figure 2$)$.

Neither PL, nor movement speed, changed over the blocks (effect of block: $p>0.4$ ). There were no differences between the last repeated block and the following random sequence ( $\mathrm{p}>$ $0.2)$.

\section{Comparison between stroke survivors and age matched controls}

There were significant differences between groups in baseline (first repetition) OT $(\mathrm{t}(18)=$ $5.455, \mathrm{p}<0.001)$ and speed $(\mathrm{t}(18)=-3.819, \mathrm{p}=0.001)$, with stroke survivors showing longer OT and slower movement speed than controls but no differences in PL $(\mathrm{p}=0.17)$. Baseline values are shown in table 2 .

\section{Insert table 2 around here}

Since there was a difference between groups for baseline OT, the OT of each block was normalised to the first repetition of the sequence to test for learning differences between groups. The normalised OT difference between the last repeated block (block 13) and the random sequence was determined as an indication of sequence specific learning.

The normalised OT difference was significantly higher for the control group than the stroke group (Mann Whitney $\mathrm{U}$ test: $\mathrm{p}=0.015$ ) indicating that sequence specific learning was impaired for the stroke survivors (figure 3). 


\section{Discussion}

This study shows that both healthy adults and stroke survivors can improve performance of an explicit movement sequence requiring gross movements of the arm. Improvement in both groups was due to faster OT with no changes in speed or accuracy of movement. However, the amount of sequence specific learning was smaller in the stroke survivors than in the controls, indicating that their ability to prepare for the next movement in the sequence was impaired.

Unlike traditional "key-based" sequence learning tasks, this paradigm is suitable for use with the paretic arm of stroke survivors with moderate and mild impairment and therefore is relevant for rehabilitation studies. All participants were more impaired (FM range 39-60) than those studied previously by Zimerman et al. [16] with their sequence learning task and so are more representative of the general stroke population. Other studies have tested stroke survivors with similar levels of impairment to those in our study, but have utilised the "unaffected", rather than the paretic hand $[8,10,11,15,18,23]$. Although some people with stroke must adapt to physical impairment by using their "unaffected" arm, the primary focus of interest for research and clinical work is to re-learn motor behaviours to improve function of the affected limb. Sequential movements are essential for many activities of daily living and therefore the results gained with this paradigm are relevant to understanding the ability of stroke survivors to re-learn and improve independence through physical therapy. Lefebvre et al. [24] used a motor learning task with stroke survivors that required similar functional movements, i.e. movement of a computer mouse with the affected hand, to examine the effect of transcranial direct current stimulation. Learning in that task was achieved by changes in 
the speed/accuracy trade-off alongside learning of the movement pattern around a maze. However, the performance of stroke survivors was not compared directly with age-matched healthy adults.

Although the sample for the current study was small, significant improvements in OT were seen for both groups without a reduction in either movement accuracy or speed. However, as noted in the introduction, two elements underlie reductions in OT; they might be due to the explicit learning of the movement sequence or to a general learning to perform the paradigm which is not sequence specific. The control group demonstrated a significantly greater difference in OT between the last block of the repeated sequence and the random sequence and so a greater sequence specific change in OT. The conclusion is that stroke can impair explicit sequence specific learning.

The reason underlying the impairment in sequence specific learning is currently unknown. It could be that the stroke group had more general learning improvements. Alternatively, sequence specific movement learning may be slower for stroke survivors than healthy controls due to reduced capacity for plasticity, with more repetitions required to achieve similar improvements in movement preparation. It could also indicate an attentional, cognitive or motor impairment due to stroke that limits the amount of learning achievable. Although the brain activation patterns during this task have not been examined, explicit sequence learning tasks have been shown to activate some motor regions more than implicit learning tasks, including premotor cortex, supplementary motor area, superior parietal lobe and thalamus [25]. The lesion locations varied between participants, but the majority had lesions overlapping at least one of these regions and also may have had impairments in connectivity between regions. 
All participants in the control group showed a longer OT for the random sequence than the last block of the repeated sequence whereas some stroke survivors did not (figure 3). One of the participants who did not show a longer OT for the random sequence had a stroke affecting the cerebellum. Boyd and Winstein [26] demonstrated that improvements in spatial accuracy with implicit learning were preserved in people with cerebellar stroke but improvements in temporal accuracy were not. Since OT is a temporal parameter, this participant's result might therefore extend their findings to suggest that temporal changes with explicit learning could also be affected by cerebellar stroke. Further, the cerebellum is known to play a role in feedforward control, which would be necessary for movement preparation to anticipate target appearance with learning. Two other participants who did not show a longer OT for the random sequence had haemorrhagic strokes affecting subcortical structures (basal ganglia and internal capsule). The putamen and thalamus have been found to be involved in both sensorimotor and sequence learning paradigms [25], and the provision of explicit information has been found to impair implicit learning in people with basal ganglia lesions [27]. Although these are all individual participants, their learning deficits may be therefore directly related to the location of their strokes. The impact of lesion location on motor sequence learning with the paretic arm requires further investigation in future studies.

Neither speed of movement nor accuracy changed with training for either group. Therefore these variables were not specifically compared across the groups. The lack of change with training may be because the focus in this explicit learning task was on the reaction to target illumination (OT) rather than movement speed and accuracy. It is also possible that path length was not sufficiently sensitive to detect very small improvements in accuracy. Stroke survivors had slower baseline movement speed than controls and may have sacrificed speed 
to focus on learning the movement sequence and maintaining accuracy. Slower movement may also be a general effect of stroke, rather than specific to the paretic arm, as previous studies with the "unaffected" hand have also reported slower performance in stroke participants than controls $[11,12,23]$. In the present study all stroke survivors used their paretic arm, regardless of previous dominance. It is not known whether the rate of sequence learning depends on hand dominance in either population.

These findings have potential implications for rehabilitation as motor learning principles underlie many physiotherapy techniques. Stroke survivors were capable of learning the movement sequence with their paretic arm, reinforcing the concept of repetitive training to improve movement preparation, but sequence specific learning was impaired. This reduced specificity of learning may be beneficial if training on one activity overlaps to other untrained movements or tasks. However, this is speculation and requires investigation.

Limitations include a small and heterogeneous sample of stroke survivors. We were interested in the effect of upper limb impairment from stroke and therefore did not restrict our sample to corticospinal lesions but rather to participants with persistent upper limb impairment. The time since stroke varied considerably across participants which may have affected participant's ability to physically perform the task due to the development of contractures or compensation strategies. Nevertheless, the results of this study provide sufficient evidence that stroke survivors exhibit impairments in motor sequence learning for this task to be used in future larger studies. Baseline OT was not matched between groups which may have influenced results. However, given than controls had a quicker baseline OT, it might be expected that they would find it more difficult to reduce it further due to a possible "ceiling effect", which was not the case. Additionally, there were a higher proportion 
of male participants in the stroke group. Men and women express differences in neuroanatomy and neurotransmitter concentration and may employ different learning strategies $[28,29]$. It is therefore possible that this gender imbalance could have contributed, in part, to the differences observed.

Of the participants recruited, not everyone was capable of completing the task, indicating that this paradigm is still not appropriate for the full range of impairments after stroke. All participants were at least six months post-stroke so it remains to be determined how motor sequence learning is affected in the acute stage when the majority of re-learning through rehabilitation takes place. Finally, we have not tested retention of learning and so cannot draw conclusions as to whether there are differences in long-term recall between groups with this paradigm.

\section{Acknowledgements}

We thank the late Roger Woledge for writing the Matlab Programme and Tony Christopher and Lindsey Marjoram for technical support.

\section{Declaration of interest}

MKF is supported by a King's College London doctoral scholarship and a project grant awarded to DJN by The Stroke Association (TSA 2013-09). The authors report no conflicts of interest. 


\section{References}

[1] Lefebvre S, Dricot L, Laloux P, Gradkowski W, Desfontaines P, Evrard F, Peeters A, Jamart J, Vandermeeren Y. Neural substrates underlying stimulation-enhanced motor skill learning after stroke. Brain 2015;138:149-63.

[2] Reis J, Robertson E, Krakauer JW, Rothwell J, Marshall L, Gerloff C, Wassermann E, Pascual-Leone A, Hummel F, Celnik PA and others. Consensus: "Can tDCS and TMS enhance motor learning and memory formation?". Brain Stimul 2008;1:363-9.

[3] Reis J, Schambra HM, Cohen LG, Buch ER, Fritsch B, Zarahn E, Celnik PA, Krakauer JW. Noninvasive cortical stimulation enhances motor skill acquisition over multiple days through an effect on consolidation. Proc Natl Acad Sci U S A 2009;106:1590-5.

[4] Dayan E, Cohen LG. Neuroplasticity subserving motor skill learning. Neuron 2011;72:443-54.

[5] Hikosaka O, Nakamura K, Sakai K, Nakahara H. Central mechanisms of motor skill learning. Curr Opin Neurobiol 2002;12:217-22.

[6] Karni A, Meyer G, Rey-Hipolito C, Jezzard P, Adams MM, Turner R, Ungerleider LG. The acquisition of skilled motor performance: fast and slow experience-driven changes in primary motor cortex. Proc Natl Acad Sci U S A 1998;95:861-8.

[7] Hubbard IJ, Parsons MW, Neilson C, Carey LM. Task-specific training: evidence for and translation to clinical practice. Occup Ther Int 2009;16:175-89.

[8] Boyd LA, Quaney BM, Pohl PS, Winstein CJ. Learning implicitly: effects of task and severity after stroke. Neurorehabil Neural Repair 2007;21:444-54.

[9] Boyd LA, Winstein CJ. Implicit motor-sequence learning in humans following unilateral stroke: the impact of practice and explicit knowledge. Neurosci Lett 2001;298:65-9.

[10] Boyd LA, Winstein CJ. Impact of explicit information on implicit motor-sequence learning following middle cerebral artery stroke. Phys Ther 2003;83:976-89.

[11] Pohl PS, McDowd JM, Filion DL, Richards LG, Stiers W. Implicit learning of a perceptual-motor skill after stroke. Phys Ther 2001;81:1780-9.

[12] Orrell AJ, Eves FF, Masters RS, MacMahon KM. Implicit sequence learning processes after unilateral stroke. Neuropsychol Rehabil 2007;17:335-54.

[13] Nissen MJ, Bullemer P. Attentional requirements of learning: Evidence from performance measures. Cognitive Psychology 1987;19:1-32.

[14] Schwarb H, Schumacher EH. Generalized lessons about sequence learning from the study of the serial reaction time task. Adv Cogn Psychol 2012;8:165-78.

[15] Dovern A, Fink GR, Timpert DC, Saliger J, Karbe H, Weiss PH, Koch I. Timing Matters? Learning of Complex Spatiotemporal Sequences in Left-hemisphere Stroke Patients. J Cogn Neurosci 2016;28:223-36.

[16] Zimerman M, Heise KF, Hoppe J, Cohen LG, Gerloff C, Hummel FC. Modulation of training by single-session transcranial direct current stimulation to the intact motor cortex enhances motor skill acquisition of the paretic hand. Stroke 2012;43:2185-91.

[17] Fugl-Meyer AR, Jaasko L, Leyman I, Olsson S, Steglind S. The post-stroke hemiplegic patient. 1. a method for evaluation of physical performance. Scand J Rehabil Med 1975;7:13-31.

[18] Dovern A, Fink GR, Saliger J, Karbe H, Koch I, Weiss PH. Apraxia impairs intentional retrieval of incidentally acquired motor knowledge. J Neurosci 2011;31:8102-8.

[19] Woodbury ML, Velozo CA, Richards LG, Duncan PW. Rasch analysis staging methodology to classify upper extremity movement impairment after stroke. Arch Phys Med Rehabil 2013;94:1527-33.

[20] Oldfield RC. The assessment and analysis of handedness: the Edinburgh inventory. Neuropsychologia 1971;9:97-113. 
[21] Stagg CJ, Jayaram G, Pastor D, Kincses ZT, Matthews PM, Johansen-Berg H. Polarity and timing-dependent effects of transcranial direct current stimulation in explicit motor learning. Neuropsychologia 2011;49:800-4.

[22] Rom D. A sequentially rejective test procedure based on a modified Bonferroni inequality. Biometrika 1990;77:663-5.

[23] Pohl PS, McDowd JM, Filion D, Richards LG, Stiers W. Implicit learning of a motor skill after mild and moderate stroke. Clin Rehabil 2006;20:246-53.

[24] Lefebvre S, Thonnard JL, Laloux P, Peeters A, Jamart J, Vandermeeren Y. Single session of dual-tDCS transiently improves precision grip and dexterity of the paretic hand after stroke. Neurorehabil Neural Repair 2014;28:100-10.

[25] Hardwick RM, Rottschy C, Miall RC, Eickhoff SB. A quantitative meta-analysis and review of motor learning in the human brain. Neuroimage 2013;67:283-97.

[26] Boyd LA, Winstein CJ. Cerebellar stroke impairs temporal but not spatial accuracy during implicit motor learning. Neurorehabil Neural Repair 2004;18:134-43.

[27] Boyd LA, Winstein CJ. Providing explicit information disrupts implicit motor learning after basal ganglia stroke. Learn Mem 2004;11:388-96.

[28] Andreano JM, Cahill L. Sex influences on the neurobiology of learning and memory. Learn Mem 2009;16:248-66.

[29] Cohen NR, Pomplun M, Gold BJ, Sekuler R. Sex differences in the acquisition of complex skilled movements. Exp Brain Res 2010;205:183-93. 
Table 1. Patient Characteristics

\begin{tabular}{|c|c|c|c|c|c|c|c|c|}
\hline Patient & Sex & Affected Arm & Dominant Arm & $\begin{array}{l}\text { Time since stroke } \\
\text { (mo) }\end{array}$ & $\begin{array}{c}\text { Age } \\
\text { (years) }\end{array}$ & $\begin{array}{c}\text { FM } \\
(\max 66)\end{array}$ & Lesion Type & Lesion Location \\
\hline 1 & M & L & $\mathrm{R}$ & 57 & 60 & 40 & I & Internal Capsule \\
\hline 2 & M & $\mathrm{R}$ & $\mathrm{R}$ & 20 & 49 & 48 & $\mathrm{H}$ & Parietal, Precentral Gyrus \\
\hline 3 & $\mathrm{~F}$ & L & $\mathrm{R}$ & 18 & 67 & 40 & I & Lacunar territory \\
\hline 4 & $\mathrm{~F}$ & L & $\mathrm{R}$ & 47 & 80 & 60 & I & Cerebellar \\
\hline 5 & M & $\mathrm{R}$ & $\mathrm{R}$ & 138 & 65 & 59 & $\mathrm{H}$ & Internal Capsule \\
\hline 6 & M & $\mathrm{R}$ & $\mathrm{R}$ & 16 & 61 & 39 & I & Pons \\
\hline 7 & M & $\mathrm{R}$ & $\mathrm{R}$ & 76 & 76 & 58 & 1 & MCA territory \\
\hline 8 & M & $\mathrm{R}$ & $\mathrm{R}$ & 118 & 66 & 39 & 1 & Pons \\
\hline $9^{a}$ & M & $\mathrm{L}$ & $\mathrm{R}$ & 27 & 57 & 35 & I & Thalamus \\
\hline $10^{\mathrm{a}}$ & $M$ & $R$ & $R$ & 11 & 74 & 57 & $\mathrm{H}$ & Basal Ganglia \\
\hline 11 & $M$ & L & L & 10 & 76 & 59 & 1 & Parietal \\
\hline 12 & $M$ & L & $R$ & 54 & 39 & 40 & $\mathrm{H}$ & $\begin{array}{c}\text { Putamen, Right lateral } \\
\text { Ventricle }\end{array}$ \\
\hline
\end{tabular}

a did not complete all 25 repetitions of task 
Motor sequence learning after stroke

Table 2. Initial values for stroke survivors and control groups

\begin{tabular}{lcc}
\hline & $\begin{array}{c}\text { Stroke } \\
\text { mean (SD) }\end{array}$ & $\begin{array}{c}\text { Control } \\
\text { mean (SD) }\end{array}$ \\
\hline OT (s) & $0.56(0.06)^{*}$ & $0.42(0.06)$ \\
PL (pixels) & $570(229)$ & $442(69)$ \\
Speed (pixels. $\left.{ }^{-1}\right)$ & $557(168)^{*}$ & $877(205)$ \\
\hline
\end{tabular}

* significant difference between groups, $\mathrm{p}<0.05$. 


\section{Figure Legends}

Figure. 1. Representation of experimental setup showing motor sequence learning programme as seen on the computer monitor. One central square and four circular targets can be seen. The circular targets were illuminated to form a 10 movement sequence. After familiarisation, 25 repetitions (13 blocks) of the repeated sequence were performed, followed by a random sequence.

Figure. 2. OT for stroke survivors (mean \pm SEM; filled circles) and controls (open squares). Block 14 (grey symbol) represents the random sequence. ${ }^{\wedge}$ and $*$ significant difference between last block of repeated sequence and random sequence for stroke survivors and controls respectively, $\mathrm{p}<0.05$.

Figure. 3. Normalised OT for the last block of the repeated sequence and the random sequence for individual participants. There was a significant difference between groups for the difference in OT $(\mathrm{p}=0.015)$ indicating reduced sequence specific learning for stroke group. 